\title{
Cord blood metabolic signatures predictive of childhood overweight and rapid growth
}

\author{
Evangelos Handakas $\mathbb{D}^{1}{ }^{1}$, Pekka Keski-Rahkonen ${ }^{2}$, Lida Chatzi ${ }^{3}$, Rossella Alfano ${ }^{1,4}$, Theano Roumeliotaki (iD ${ }^{5}$, Michelle Plusquin ${ }^{4}$, \\ Léa Maitre ${ }^{6,7,8}$, Lorenzo Richiardi ${ }^{9}{ }^{9}$, Sonia Brescianini ${ }^{10}$, Augustin Scalbert ${ }^{2}$, Nivonirina Robinot ${ }^{2}$, Tim Nawrot ${ }^{4}$, Franco Sassi ${ }^{11}$, \\ Martine Vrijheid (iD) ${ }^{6,7,8}$, Paolo Vineis ${ }^{1}$ and Oliver Robinson (iD) ${ }^{1 凶}$
}

(c) The Author(s) 2021

INTRODUCTION: Metabolomics may identify biological pathways predisposing children to the risk of overweight and obesity. In this study, we have investigated the cord blood metabolic signatures of rapid growth in infancy and overweight in early childhood in four European birth cohorts.

METHODS: Untargeted liquid chromatography-mass spectrometry metabolomic profiles were measured in cord blood from 399 newborns from four European cohorts (ENVIRONAGE, Rhea, INMA and Piccolipiu). Rapid growth in the first year of life and overweight in childhood was defined with reference to WHO growth charts. Metabolome-wide association scans for rapid growth and overweight on over 4500 metabolic features were performed using multiple adjusted logistic mixed-effect models and controlling the false discovery rate (FDR) at $5 \%$. In addition, we performed a look-up analysis of 43 pre-annotated metabolites, previously associated with birthweight or rapid growth.

RESULTS: In the Metabolome-Wide Association Study analysis, we identified three and eight metabolites associated with rapid growth and overweight, respectively, after FDR correction. Higher levels of cholestenone, a cholesterol derivative produced by microbial catabolism, were predictive of rapid growth $\left(p=1.6 \times 10^{-3}\right)$. Lower levels of the branched-chain amino acid (BCAA) valine $\left(p=8.6 \times 10^{-6}\right)$ were predictive of overweight in childhood. The area under the receiver operator curve for multivariate prediction models including these metabolites and traditional risk factors was 0.77 for rapid growth and 0.82 for overweight, compared with 0.69 and 0.69 , respectively, for models using traditional risk factors alone. Among the 43 pre-annotated metabolites, seven and five metabolites were nominally associated $(P<0.05)$ with rapid growth and overweight, respectively. The BCAA leucine, remained associated $\left(1.6 \times 10^{-3}\right)$ with overweight after FDR correction.

CONCLUSION: The metabolites identified here may assist in the identification of children at risk of developing obesity and improve understanding of mechanisms involved in postnatal growth. Cholestenone and BCAAs are suggestive of a role of the gut microbiome and nutrient signalling respectively in child growth trajectories.

International Journal of Obesity (2021) 45:2252-2260; https://doi.org/10.1038/s41366-021-00888-1

\section{INTRODUCTION}

Childhood obesity has become a global epidemic in developed as well as in developing countries [1], with significant long-term consequences on both physical and psychological health, social and economic outcomes [2]. Behavioural dimensions such as diet and physical activity, and an 'obesogenic environment' that shapes those behaviours, have contributed to the spread of childhood obesity $[3,4]$. In the last decades, there has been a growing interest in the idea that the early life environment can have lasting effects on the physiology and metabolism of the fetus and is associated with the early metabolic programming of human health [5-7]. Recent studies have revealed that several in utero exposures such as maternal socioeconomic status, clinical and environmental factors are associated with growth in infancy and with the subsequent development of childhood overweight or obesity [8-13]. The prenatal environment can affect fetus weight homeostasis and may result in a 'thrifty phenotype' that stores excess calories and predisposes children to weight gain [14]. Hence, a metabolic signature at birth may help elucidate the mechanisms involved in metabolic health later in life.

\footnotetext{
${ }^{1}$ Medical Research Council Centre for Environment and Health, School of Public Health, Imperial College London, London, UK. ${ }^{2}$ Nutrition and Metabolism Section, International

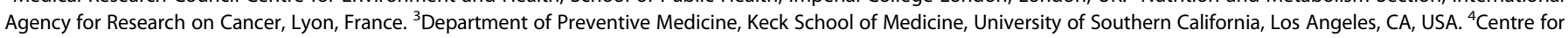

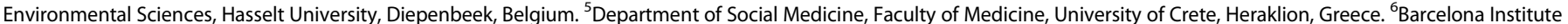

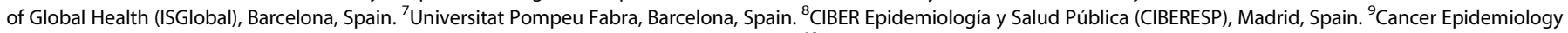

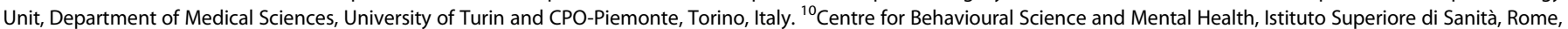
Italy. ${ }^{11}$ Centre for Health Economics \& Policy Innovation, Department of Economics \& Public Policy, Imperial College Business School, South Kensington Campus, London, UK. email: o.robinson@imperial.ac.uk
}

Received: 20 November 2020 Revised: 30 April 2021 Accepted: 22 June 2021

Published online: 12 July 2021 
Metabolomics, the profiling of circulating small molecules, has been increasingly applied to investigate biological mechanisms associated with childhood obesity $[15,16]$. However, few studies have investigated metabolic changes in cord blood that may predict subsequent infant growth and overweight and obesity [17]. Isganaitis, Rifas-Shiman et al. [18] analysed the metabolome in cord blood plasma from 26 cases and 26 controls differing in their postnatal weight trajectories using targeted mass spectrometry (MS) analysis of 415 metabolites, nested in an American cohort. There was a trend for lower levels of tryptophan metabolites in children that followed a rapid growth to obesity at 7 years trajectory. Sorrow, Maguire et al. [19] similarly applied a targeted MS analysis of 384 metabolites in cord blood of 25 obese and non-obese American children at 3-5 years. Children with obesity had elevated lipid species, acetaminophen metabolites and acylcarnitines compared with non-obese children, although no multiple testing correction was applied. Hellmuth, Uhl et al. [20] applied a range of targeted LC-MS assays to assess 209 metabolites in cord blood of 700 German children in relation to birthweight, postnatal weight gain and BMI throughout adolescence. Although many metabolites were associated with weight at birth, no associations with postnatal measures survived multiple testing correction. Although initial studies have so far been based on small numbers of children or limited numbers of molecules, they reveal the potential of metabolic profiling in detecting biomarkers and pathways related to rapid growth in infancy as well as to overweight and obesity in early childhood. Identifying markers that are predictive of obesity onset may assist in the development of targeted intervention programmes for at-risk groups of children.

In this study, we have investigated the cord blood metabolic signatures of rapid growth in infancy and overweight in early childhood in four European birth cohorts, using untargeted LCMS-based metabolic profiling. Our aims were twofold: firstly, to identify markers associated with rapid growth and overweight risk to provide mechanistic insight and elucidate causal pathways to obesity; and secondly to improve prediction of obesity risk in neonates through assessment of the predictive performance of models incorporating identified metabolites, in comparison with models based on traditional risk factors alone.

\section{MATERIALS AND METHODS Study population}

The study population included participants from four population-based birth participating in the STOP project: ENVIRONAGE [21] (Belgium), INMA [22] (Spain), Piccolipiu [23] (Italy) and Rhea [24] (Greece). Ethical approval was obtained from the local Research Ethics Committees from each centre. Informed consent was obtained from the parents of the children. Further details of blood sampling, clinical, dietary and socioeconomic data of cohort individuals are given in the respective references and supporting information 1.

\section{Untargeted metabolomics}

Cord blood samples were analysed in randomised order as a single uninterrupted batch with a UHPLC-QTOF-MS system (Agilent Technologies), as previously described [25]. Further details of the acquisition and structural annotation of features are given in supporting information 1.

\section{Outcome assessment}

Rapid growth in infants in the first 12 months was categorised based on the definition of Ong et al. [25]. According to this definition, a clinically significant increment that indicates rapid growth occurs when there is a gain in weight of at least 0.67 standard deviations between different target ages. In this study, length data at birth were not available. Hence, rapid growth was defined as the weight $z$ score change of $>0.67$ standard deviations (SD) between birth and twelve months of age based on World Health Organisation (WHO) growth charts [26]. A two-step prediction approach was used for calculating sex- and age-specific weight at exactly
12 months, using fractional polynomials of age by gender in each cohort [27] (supporting information 1).

To maintain sample size for the analysis of overweight in early childhood, we used a single measurement at an age greater than four years and as close to 6 years as available. The classification for healthy and overweight was based on WHO sex-adjusted and age-adjusted BMI $z$ scores. WHO provides different classifications scheme for children under the age of 5 years ( $0-5<$ years) [28] and over the age of 5 years (5-18 years) [29]. Following the WHO proposed classification by De Onis and Lobstein [30], children younger than 5 years were classified as overweight if they had a BMI $z$ scores $>1$ SD and children over 5 years were classified as overweight if they had a BMI z-score greater than 2 SDs [30].

\section{Statistical analysis}

A Metabolome-Wide Association Study (MWAS) was applied to investigate the association between cord blood metabolomics and infant rapid growth/childhood overweight using multiple mixed-effect logistic regression models using the Ime4 R package [31]. The basic model (Model 1) was adjusted for sex and age of the child at outcome measurement, ethnicity and we used a random-effect for cohort. To account for multiple testing, a Benjamini-Hochberg false discovery rate (FDR) [32] was applied using a cutoff of $5 \%$.

We then applied additional covariate adjustment to significant features identified in the MWAS analysis. A directed acyclical graph was used to visualise assumptions regarding covariates for further model adjustment (Figure S1). Covariates were chosen based on a bivariate analysis of their correlation with outcomes (Logistic Regression). The resulting model (Model 2) included Model 1 covariates and maternal BMI, paternal BMI, gestational age, weight gained during pregnancy, paternal education, passive and active smoking status during pregnancy, parity and mode of delivery.

Pathway enrichment analysis on significant features was conducted using the Mummichog programme [33], supplemented with manual curation of the metabolite identities assigned by Mummichog (supporting information 1).

A look-up analysis, using the same statistical approach as the MWAS analysis (including 5\% FDR), was conducted on 43 metabolites that had been previously annotated in the same data set as used in this study, due to their associations with birthweight $[34,35]$ or because they had previously been reported to predict a rapid growth leading to overweight in childhood trajectory, and could also be identified with high confidence through retention time and MS/MS matching in our data set [18].

In sensitivity analyses, we re-ran Model 2 for metabolites associated with rapid growth or overweight, stratified by cohort, sex and size for gestational age and additionally adjusted for birthweight.

We further assessed how well rapid growth in infancy or overweight in early childhood are predicted using metabolites in comparison with traditional factors using Random Forest classification models [36] (supporting information 1). We used three different sets of variables for each of the outcomes: (1) traditional risk factors (sex, birthweight, ethnicity, maternal BMI, paternal BMI, gestational age, maternal weight gain during pregnancy, paternal education, maternal passive and active smoking status during pregnancy, parity and mode of delivery), (2) significantly associated metabolites from the MWAS analysis and (3) significantly associated metabolites from MWAS analysis in combination with traditional risk factors. A bootstrap method of 1000 repetitions was advocated to quantify optimism and evaluate the generalisation of the model. A threefold crossvalidation routine was performed on the training set (random $80 \%$ of the total observations) to each model to determine the optimum probability threshold. The model performance was evaluated on the relevant test set (remaining $20 \%$ of the total observations) using receiver operating characteristic (ROC curve) and area under the curve or AUROC for assessing the goodness-of-fit of the classifier. To further evaluate the predictive model, we performed a leave-one-out analysis by repeating the modelling process on a combined data set with one cohort retained as the validation set (supporting information 1).

\section{RESULTS}

\section{Participant Information and metabolomic data}

Table 1 shows the characteristics of the population used in the analysis of rapid growth in infancy and overweight in early childhood (stratification by cohort, including available dietary information, is presented in Table S1, S2. In bivariate analyses 
Table 1. Demographic, anthropometric and clinical outcome variables.

\begin{tabular}{|c|c|c|c|c|c|c|}
\hline & \multicolumn{3}{|c|}{ Rapid growth at 12 months analysis } & \multicolumn{3}{|c|}{$\begin{array}{l}\text { Overweight/obesity at early childhood } \\
\text { analysis }\end{array}$} \\
\hline & $(n=391)$ & Missing & $p$ value & $(n=272)$ & Missing & $p$ value \\
\hline \multicolumn{7}{|l|}{ Cohort } \\
\hline RHEA & $100(25.6 \%)$ & & & 97 (35.7\%) & & \\
\hline ENVIRONAGE & $109(27.9 \%)$ & & & $-b$ & & \\
\hline Piccolipiu & $95(24.3 \%)$ & & & $79(29.0 \%)$ & & \\
\hline Gender & & $0(0 \%)$ & 0.910 & & $0(0 \%)$ & 0.801 \\
\hline Male & $204(52.2 \%)$ & & & $145(53.3 \%)$ & & \\
\hline Female & $187(47.8 \%)$ & & & $127(46.7 \%)$ & & \\
\hline Birthweight (grams) & & $0(0 \%)$ & $3.34 \mathrm{E}-15$ & & $0(0 \%)$ & 0.191 \\
\hline Mean (SD) & $3295(445)$ & & & $3265(412)$ & & \\
\hline Maternal active smoking & & $1(0.3 \%)$ & 0.608 & & $2(0.7 \%)$ & 0.045 \\
\hline No & $324(82.9 \%)$ & & & $216(79.4 \%)$ & & \\
\hline Yes & $66(16.8 \%)$ & & & $54(19.9 \%)$ & & \\
\hline Maternal passive smoking & & $8(2.0 \%)$ & 0.153 & & $6(2.2 \%)$ & 7.10E-05 \\
\hline No & $233(59.6 \%)$ & & & $123(45.2 \%)$ & & \\
\hline Yes & $150(38.4 \%)$ & & & $142(52.2 \%)$ & & \\
\hline Maternal BMI $\left(\mathrm{kg} / \mathrm{m}^{2}\right)$ & & $1(0.3 \%)$ & 0.392 & & $1(0.4 \%)$ & 7.93E-04 \\
\hline Mean (SD) & $23.9(4.58)$ & & & $23.7(4.51)$ & & \\
\hline Maternal weight gain (kilograms) & & $12(3.1 \%)$ & 0.021 & & $11(4.0 \%)$ & 0.090 \\
\hline Mean (SD) & $13.6(5.16)$ & & & $13.3(5.09)$ & & \\
\hline No & $35(9.0 \%)$ & & & $13(4.8 \%)$ & & \\
\hline Yes & 354 (90.5\%) & & & $259(95.2 \%)$ & & \\
\hline Father's education & & $13(3.3 \%)$ & & & $3(1.1 \%)$ & \\
\hline Primary school & $70(17.9 \%)$ & & 0.296 & $58(21.3 \%)$ & & 0.202 \\
\hline Secondary school & $189(48.3 \%)$ & & 0.282 & $138(50.7 \%)$ & & 0.019 \\
\hline University or higher & $119(30.4 \%)$ & & & $73(26.8 \%)$ & & \\
\hline Paternal BMI $\left(\mathrm{kg} / \mathrm{m}^{2}\right)$ & & $7(1.8 \%)$ & 0.139 & & $3(1.1 \%)$ & $1.09 \mathrm{E}-04$ \\
\hline Mean (SD) & $25.8(3.46)$ & & & $26.0(3.62)$ & & \\
\hline Rapid growth & & $0(0 \%)$ & & & $12(4.4 \%)$ & $1.13 \mathrm{E}-03$ \\
\hline No & $280(71.1 \%)$ & & & $168(61.8 \%)$ & & \\
\hline Yes & $114(28.9 .0 \%)$ & & & $92(33.8 \%)$ & & \\
\hline Overweight/obesity in childhood & & - & & & $0(0 \%)$ & \\
\hline No & - & & & $224(82.4 \%)$ & & \\
\hline Yes & & & & $48(17.6 \%)$ & & \\
\hline Age at childhood BMI measurement (years) & - & - & & & $0(0 \%)$ & \\
\hline Mean (SD) & - & - & & $5.43(1.00)$ & & \\
\hline
\end{tabular}

Values are given in mean (standard deviation, SD) or percent (\%).

${ }^{a} p$ value for association with rapid growth at 12 months of age and overweight in early childhood calculated from logistic regression.

${ }^{b}$ ENVIRONAGE was not included in the analysis of overweight in childhood as follow-up assessment was only available until 2 years.

CThe classification for healthy and overweight was based on WHO sex-adjusted and age-adjusted BMI z scores. 


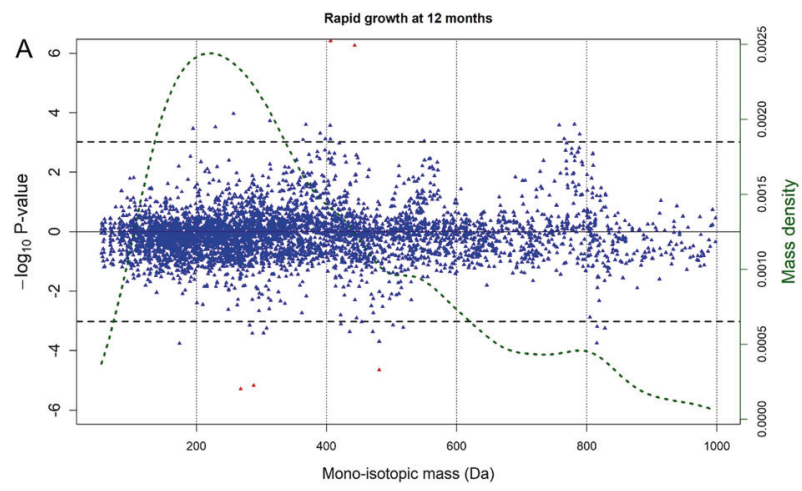

Overweightobesity in early childhood

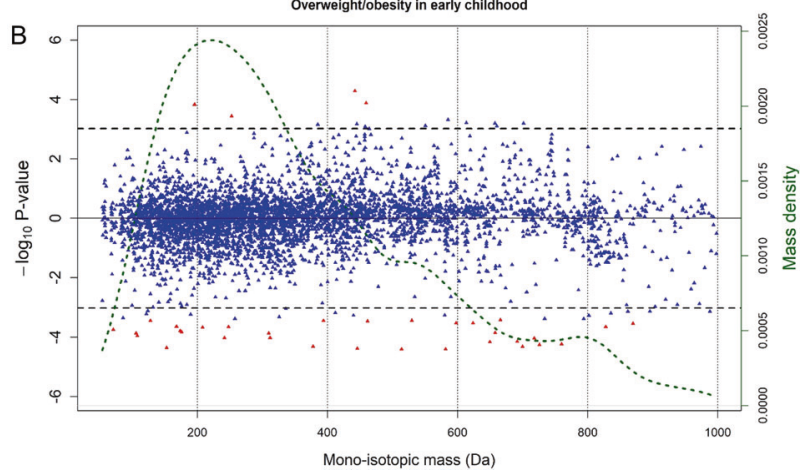

Fig. 1 Metabolome wide associations with rapid growth and overweight. Signed Manhattan-type plot presenting the analysis of the 4714 UPLC-MS metabolic features for Model 1 for A rapid growth at twelve months of age and for B overweight in early childhood. The red dots represent the features that remain significant after applying the FDR threshold of $5 \%$, whereas blue dots do not. The vertical axis shows the signed $-\log 10 P$ value. The horizontal axis represents the monoisotopic mass (in Da). UPLC-MSassociated metabolic features are available in Table S3 and Table S6. The dotted green line represents the mass density.

(Table 1), birthweight, parity, maternal weight gained during pregnancy, mode of delivery and gestational age were all significantly associated $(P<0.05)$ with rapid growth, while maternal passive and active smoking during pregnancy, maternal $\mathrm{BMI}$, paternal education level, paternal BMI and rapid growth in infancy were significantly associated with overweight in early childhood. After data filtering procedures, 4714 metabolic features were available for statistical analysis.

\section{Cord blood metabolomics and rapid growth in the first year of life}

The analysis of rapid growth included 391 children, with 114 $(28.9 \%)$ classed as rapid growers in the first year of life. In MWAS analysis, adjusting for age at the outcome measurement, sex, cohort and ethnicity (Model 1), six metabolic features were significantly associated (FDR $<5 \%$ ) with rapid growth in the first year of life (Fig. 1A). Table S3 contains the retention time as well as the exact mass of all significantly associated features, including unassigned metabolites. The metabolic features were grouped into four metabolites after grouping of ions originating from the same molecule (matched by retention time and pairwise feature correlation, Table S3). One metabolite could be identified as cholestenone (4-cholesten-3-one; HMDB0000921), a steroid lipid in the class of cholesterols. Upon adjustment for further covariates (Model 2), three of the four associated metabolites, including cholestenone, remained significantly associated with rapid growth (Fig. 2A). Cholestenone levels were higher in the cord blood of rapid growers, whereas levels of the rest of the metabolites were lower in the cord blood of rapid growers.
In a look-up analysis, we analysed associations with 43 known metabolites (retention time and $\mathrm{m} / \mathrm{z}$ information given in Table S4) in the metabolome data set that had been previously annotated based on their associations with birthweight [34, 35], or with rapid infancy weight gain and childhood obesity [18] (including indolelactic acid, sphingosine, tryptophan and leucine) (Table S5). Fourteen metabolites were associated with rapid growth in the first year of life (Fig. 2B) after correcting for 5\% FDR in basic adjustment analyses (Model 1), including higher levels of nine phosphatidylcholines (PCs) or LysoPCs, cholestenone, cholesterol, progesterone and two acylcarnitines tetradecadiencarnitine (C14:2) and decenoylcarnitine (C10:1). In additionally adjusted analyses (Model 2) cholestenone, two PCs (PC(34:2) and plasmalogen $\mathrm{PC}(36: 4) / P C(0-36: 5)$ ), two acylcarnitines, docosahexaenoic acid (DHA), diacylglycerol (C36:4) and progesterone were nominally associated $(\mathrm{P}<0.05)$ with rapid growth (Fig. 2B). Directions of association with rapid growth were opposite to directions observed previously with birthweight [34]. Correcting Model 2 for $5 \%$ FDR, only cholestenone remained associated with rapid growth in the first year of life.

As shown in the network graph (Fig. 2C), cholestenone was highly correlated with $\mathrm{PC}(34: 2)$, moderately correlated with unidentified metabolite U4 and had weaker, positive correlations with the other rapid growth-associated metabolites. We noted strong correlations between $\mathrm{DHA}$ and plasmalogen $\mathrm{PC}(36: 4) / \mathrm{PC}(\mathrm{O}-$ $36: 5)$ as well as between tetradecadiencarnitine (C14:2) and PC $(34: 2)$.

Mummichog analysis indicated enrichment among rapid growers in the 'C21-steroid hormone biosynthesis and metabolism' and 'Androgen and oestrogen biosynthesis and metabolism' pathways, with weaker support for enrichment of the 'Urea cycle/ amino group metabolism' pathway (supporting information 1 and 2, Table S10).

Cord blood metabolomics and overweight in early childhood The analysis of child overweight in early childhood included 272 children from the Piccolipiu, Rhea and INMA cohorts, of which 48 $(17.6 \%)$ were classed as being overweight or obese (mean age at weight status assessment: 5.12 years (SD:1.11)). In the MWAS, adjusting for cohort and ethnicity (Model 1), 36 features were significantly associated (FDR $<5 \%$ ) with overweight in early childhood (Fig. 1B). After grouping ions originating from the same compound (Table S6), there were eight unique compounds associated with overweight (Fig. 3A). One compound could be annotated as valine, a branched-chain amino acid. Retention time as well as exact mass of all significantly associated features, including unassigned compounds, are available in Table S6. The inverse association of valine with overweight was strengthened upon additional covariate adjustment (Model 2) and remained significant after FDR correction.

In an analysis of the 43 pre-annotated metabolites, leucine and DHA were nominally associated $(P<0.05)$ with overweight in basic analyses (Model 1) (Fig. 2B). In additionally adjusted analyses (Model 2) lower levels of leucine, progesterone, indolelactic acid, hexenoylcarnitine (C6:1), hexadecenoylcarnitine (C16:1) and DHA were nominally associated $(P<0.05)$ with overweight in early childhood (Table S7). Directions of association with overweight were consistent with directions observed previously with birthweight [34]. Only leucine, a BCAA previously identified in relation to rapid infancy weight gain and childhood obesity by Isganaitis, Rifas-Shiman et al. [18], remained significant after FDR correction.

Valine was moderately correlated with DHA and had weaker correlations with the unidentified compounds U4, U5 and U7 and stronger correlations with U3 and hexadecenoylcarnitine (C16:1). Leucine had a weak negative correlation with Valine and strong negative correlations with U1, U4, U5 and U7. Strong correlations were observed between progesterone and indolelactic acid as well as between the compounds U1, U4, U5 and U7 (Fig. 3C). 


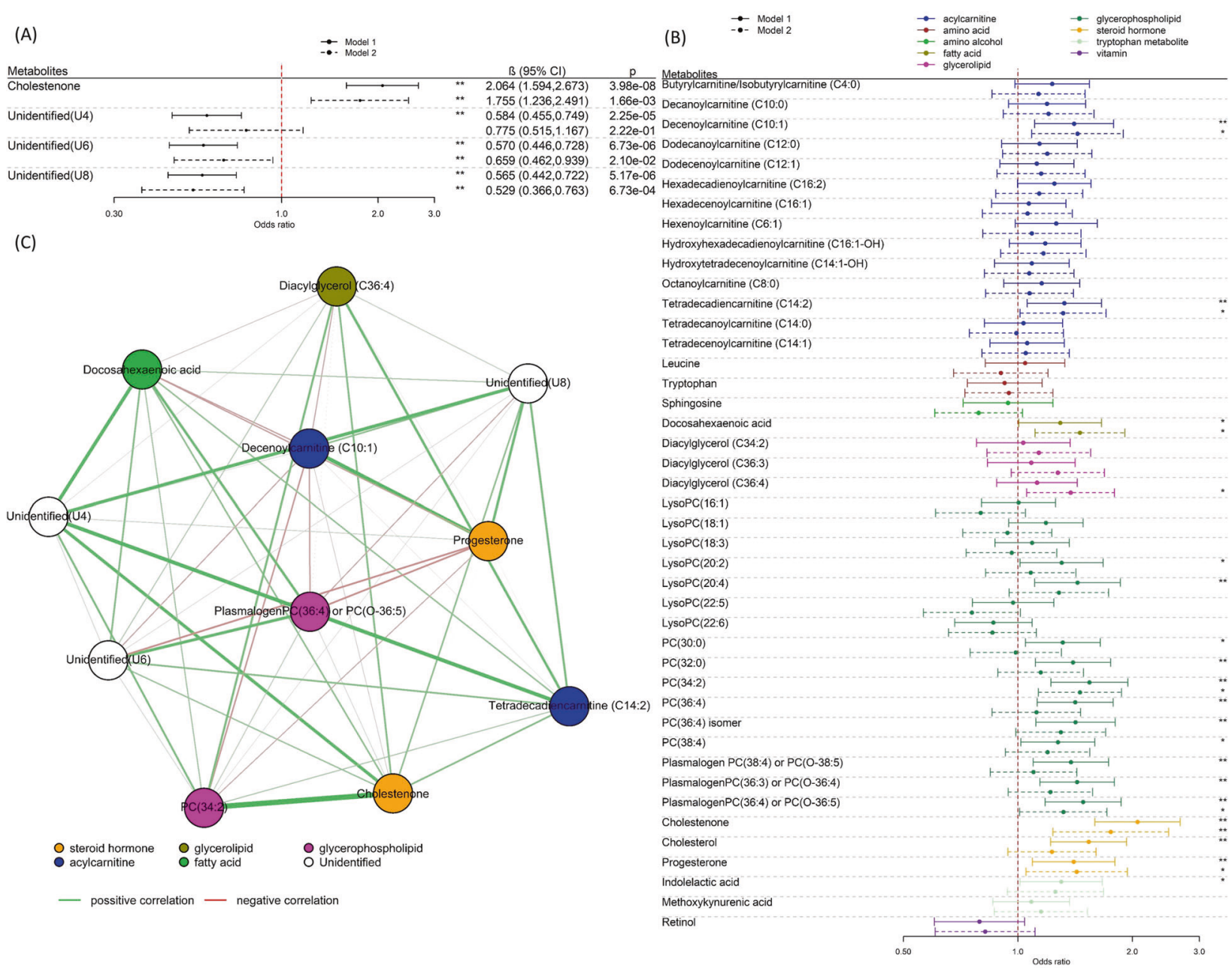

Fig. 2 Metabolite associations with rapid growth. A Regression coefficients per standard deviation (95\% confidence interval) between features and rapid growth at 12 months across all four cohorts $(N=391)$, identified in MWAS analysis. B Regression coefficients per standard deviation (95\% confidence interval) between 43 pre-annotated metabolites and rapid growth at twelve months across all four cohorts $(N=$ 391). The solids lines represent the results of Model 1 (adjusted for cohort and ethnicity) and the dotted lines the results of Model 2 (Model 1 further adjusted for maternal BMI, paternal BMI, gestational age, weight gained during pregnancy, paternal education passive and active smoking status during pregnancy, parity as well as a delivery mode). The ${ }^{*}$ declares $P<0.05$ while ${ }^{* * F D R}<0.05$. C Network graph (Pearson correlations) of metabolites associated with rapid growth at 12 months of age.

Mummichog analysis did not provide strong support for enrichment of specific pathways with childhood overweight (supporting information 1 and 3, Table S11).

\section{Multivariate prediction models}

We next utilised Random Forest classification models to evaluate the predictive performance of three different input variable sets for each of the two outcomes (Fig. 4). The rapid growth prediction model trained using only traditional risk factors exhibited a moderate predictive ability of an AUROC value of 0.69 (bootstrap $95 \%$ confidence interval (Cl):0.62-0.77) (Table S8). Adding the four metabolites (cholestenone, U2, U4 and U8) identified in the MWAS analysis into the prediction model, increased the AUROC to 0.77 (bootstrap 95\% Cl: 0.73-0.81) (Fig. 4A). For overweight, using traditional risk factors alone, the AUROC was 0.69 (bootstrap 95\% $\mathrm{Cl}$ : 0.63-0.75), while a model using only the eight metabolites, Valine, U1, U2, U3, U4, U5, U7 and U9, identified in the MWAS analysis had an AUROC of 0.77 (bootstrap 95\% Cl: 0.73-0.81) (Table S8). The combined traditional risk factor and metabolite model was strongly predictive of overweight with an AUROC of 0.82 (bootstrap 95\% Cl: 0.79-0.85) (Fig. 4B). The leave cohort out analysis also showed improvement in predictive performance using metabolites, in the majority of cohorts (Table S9).

\section{Sensitivity analysis}

To assess the robustness and consistency of our results, we stratified our population by cohort and by sex and repeated the adjusted models (Model 2) across each subpopulation. Regarding rapid growth, results were generally consistent across cohorts for all identified metabolites, including cholesterone (Figure S2). However, opposite directions of effects were observed in the Piccolipiu cohort for $\mathrm{PC}(34: 2)$ and plasmalogen $\mathrm{PC}(36: 4) / \mathrm{PC}(\mathrm{O}-$ 36:5). Regarding overweight, results were again consistent across cohorts (Figure S3), although wide confidence intervals were observed in Piccolipiu (related to the small number of overweight cases available in this cohort). For valine, strong associations were noted in both the INMA and Rhea cohorts. For rapid growth, stronger associations were observed in boys with $\mathrm{PC}(34: 2)$ and diacylglycerol (C36:4), while in girls stronger associations with rapid growth were observed with progesterone, tetradecadiencarnitine (C14:2), decenoylcarnitine(C10:1) and DHA (Figure S4). Very similar associations were seen with overweight upon stratification by sex (Figure S5).

To assess the role of birthweight in observed associations, we additionally adjusted our models for birthweight. There was some attenuation in effect size in associations for rapid growth (Figure S6), however, the attenuation with cholestenone was modest and 


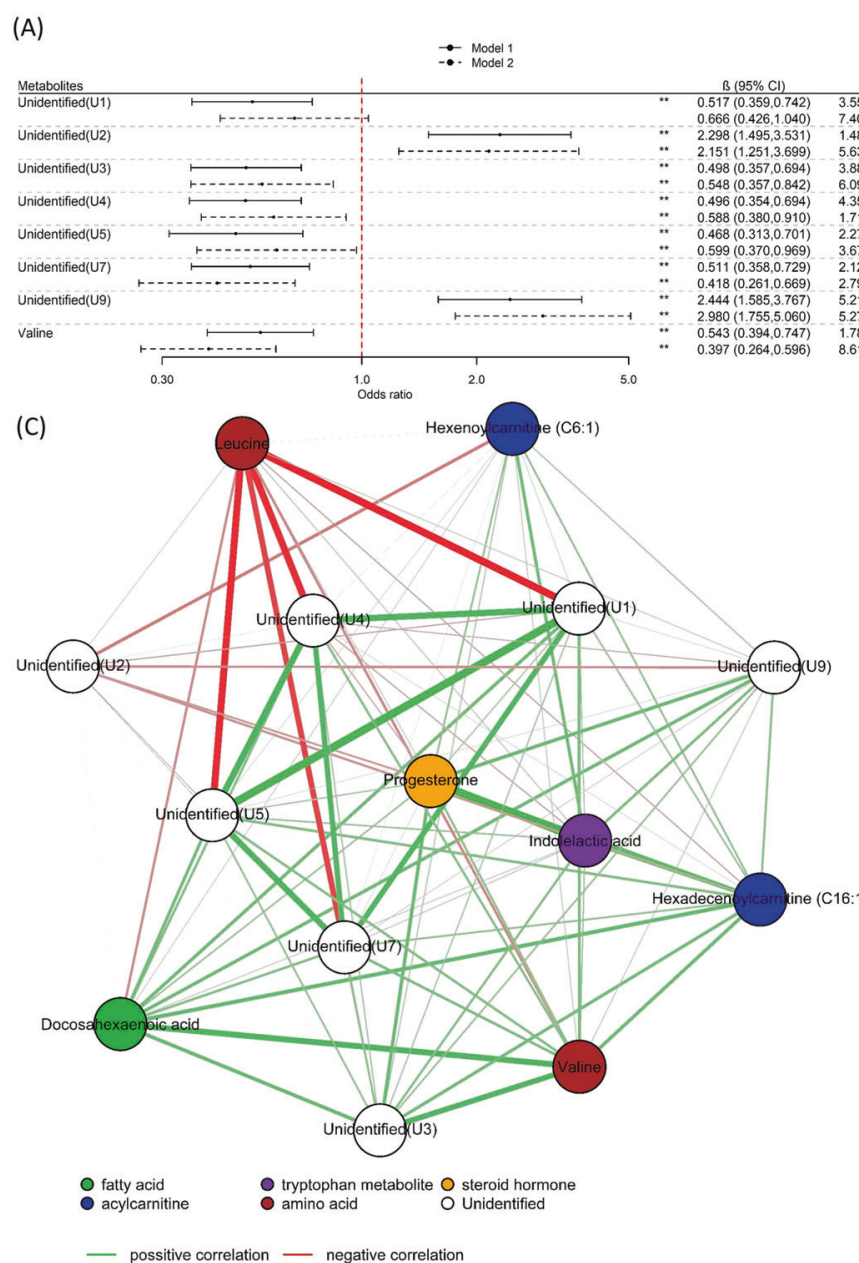

(B) $\quad \therefore$ Model 1

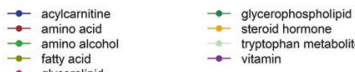

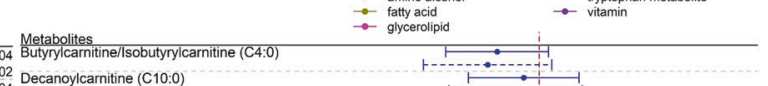
$400-02$ Decanoylcarnitine (C10:0)
48.04
$63 \mathrm{e}-03$
Decenoylcarnitine (C10:1) $78 \mathrm{e}-04$ Hydroxytetradecenoylcarnitine $(\mathrm{C} 14: 1-\mathrm{OH})$
$861 \mathrm{e}-06$ Octanoylcarnitine (C8:0) Tetradecanoylcarnitine (C14:0) Tetradecenoylcarnitine (C14:1) Leucine Tryptophan Sphingosine Docosahexaenoic acid Diacyiglycerol (C34:2) Diacylglycerol (C36:3) Diacyiglycerol (C36:4) LysoPC (16:1) LysoPC (18:1) LysoPC (18:1) LysoPC (18:3)
LysoPC (20:2) LysoPC (20:4) LysoPC (22:5) LysoPC(22:5) ysoPC(220) $\mathrm{PC}(30: 0)$ $\mathrm{PC}(32: 0)$ $\mathrm{PC}(34: 2)$ $\mathrm{PC}(36: 4)$ $\mathrm{PC}(36: 4)$ isomer $\mathrm{PC}(38: 4)$ Plasmalogen $\mathrm{PC}(38: 4)$ or $\mathrm{PC}(\mathrm{O}-38: 5)$ Plasmalogen $\mathrm{PC}(36: 3)$ or $\mathrm{PC}(\mathrm{O}-36.4)$ Plasmalogen $\mathrm{PC}(36: 4)$ or $\mathrm{PC}(0-36.5)$ Cholestenone Cholesterol Progesterone Indolelactic acid Methoxykynurenic acid Retinol

Fig. 3 Metabolite associations with overweight. A Regression coefficients per standard deviation (95\% confidence interval) between features with overweight in early childhood $(N=272)$, identified in MWAS analysis. B Regression coefficients per standard deviation $(95 \%$ confidence interval) between 43 pre-annotated metabolites with overweight in early childhood $(N=272)$. The solids lines represent the results of Model 1 (adjusted for age of child at outcome measurement, cohort and ethnicity) and the dotted lines the results of Model 2 (Model 1 further adjusted for maternal BMI, paternal BMI, gestational age, weight gained during pregnancy, paternal education passive and active smoking status during pregnancy, parity as well as delivery mode). The ${ }^{*}$ declares $P<0.05$ while ${ }^{*}$ FDR $<0.05$. C Network graph (Pearson correlations) of metabolites associated in early childhood.

significance was retained. Adjustment for birthweight had little effect on associations with overweight (Figure S7). Upon stratification by size for gestational age $(<$ and $\geq 33$ rd percentile of birthweight for gestational age, Figure S8) we observed stronger associations with cholestenone and rapid growth as well as DHA and rapid growth among larger for gestational age ( $\geq 33 \mathrm{rd}$ percentile) infants. We noted stronger associations with hexadecenoylacarnitine (C16:1), hexenoylcarnitine(C6:1), leucine and valine and overweight among smaller for gestational age $(<33 \mathrm{rd}$ percentile) infants (Figure S9).

\section{DISCUSSION}

This is the first study to date that investigates the association between untargeted metabolic profiles of cord blood and rapid growth at the first year of life and overweight/obesity in early childhood. We identified cholestenone and BCAA levels in cord blood as predictive of rapid growth and overweight/obesity, respectively, among healthy deliveries from four European populations. In multivariate analysis, we found that the addition of metabolites substantially improved prediction of both rapid growth and overweight compared with models using traditional risk factors alone.
Higher levels of cholestenone were identified as predictive of rapid growth in the MWAS analysis, with consistent effects noted across the four included cohorts. Little is known about the effects of cholestenone on health. It has previously been reported to be associated with CPG sites that are differentially methylated in relation to birthweight [35], however, birthweight did not appear to be an important contributor to the relationship between cholestenone and rapid growth in our study. Supplementation of diet with cholestenone leads to growth retardation in rodents and high levels cause hypertrophy of the adrenal glands, which may suggest potential endocrine effects [37, 38]. Cholestenone is produced by bacterial catabolism of cholesterol in the intestinal tract [39]. It therefore may be serving as a proxy indicator of the relative abundance of various microbiota present at birth, although the infant gut microbiome is generally uniform and under-developed at this stage [40]. Indeed, gestational age, which is known to influence the composition of the neonatal gut microbiome [41], was strongly associated with cholestenone levels in our data. However, the strong association between cholestenone and rapid growth remained after adjustment for gestational age. The role of the gut microflora in obesity is increasingly recognised [42] and differences in faecal microbiota composition 

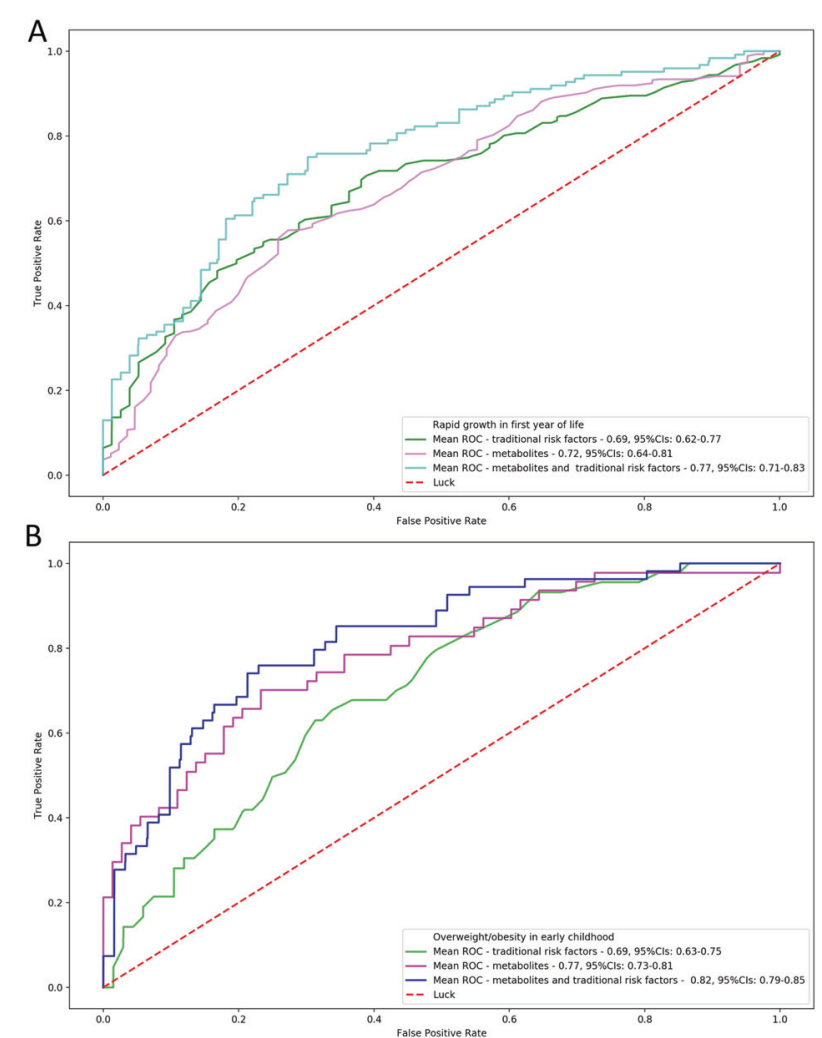

Fig. 4 Multivariate prediction models of rapid growth and overweight. ROC mean value of 1000 bootstrapped model of threefolds for $\mathbf{A}$ rapid growth at 12 months of age after grouping the ions (nine metabolites) (population size: $N=391$ ) and B overweight throughout early childhood (population size: $N=272$ ).

measured during the first year of life have been found to be associated with weight status in later childhood [43].

Lower levels of the BCAAs valine and leucine were associated with overweight/obesity in early childhood, with consistent effects across both the Rhea and INMA cohorts. Associations were somewhat stronger with valine than leucine. Lower levels of cord blood leucine were also identified as nominally associated with children on a rapid growth trajectory by the study of Isganaitis, Rifas-Shiman et al. [18]. This is in contrast with the study of Hellmuth et al., where no associations were reported between BCAAs in cord blood and weight status at 2 and 10 years, although the authors speculated that the long storage period in their study may have degraded certain metabolites such as amino acids. BCAAs levels in cord blood represent the balance of supply, from the mother and from protein degradation, and of clearance through protein synthesis, excretion and BCAA catabolism and/or oxidation. BCAAs have a complex relationship with overweight and obesity. On one hand, higher levels in blood are consistently associated with obesity, insulin resistance and type 2 diabetes. Adjustment for maternal BMI, which would be expected to increase maternal levels and the fetal supply of BCAAs, strengthened the association between cord blood BCAA levels and childhood overweight, suggesting some negative confounding. On the other hand, numerous intervention studies and animal studies have shown that increasing dietary intake of BCAAs has beneficial signalling effects, with positive effects on parameters including body composition, glycemia and satiety [44]. Multiple mechanisms for these positive effects have been proposed including direct effects on hypothalamic and brainstem processes involved in satiety [44]. Cord blood BCAAs levels could therefore influence later propensity for overweight through causal processes such as control of food intake or alternatively serve as a marker of other metabolic processes that influence both propensity for weight gain and levels of BCAAs.

Apart from the association between leucine and overweight, no other associations were observed for metabolites identified by Isganaitis, Rifas-Shiman et al. [18]. Among metabolites previously identified as associated with birthweight, we identified higher levels of progesterone, $\mathrm{PC}(34: 2)$, plasmalogen $\mathrm{PC}(36: 4) / \mathrm{PC}(\mathrm{O}-36: 5)$, DHA, decenoylcarnitine (C10:1), tetradecadiencarnitine (C14:2) and diacylglycerol (C36:4) as nominally associated with rapid growth, although these did not pass multiple testing correction. Progesterone is the major progestational hormone involved throughout all stages of pregnancy, and the pathway enrichment analysis also highlighted the role of hormonal signalling in rapid growth. DHA supplementation in milk has been shown to increase growth among preterm infants [45]. For overweight in early childhood, we noted nominal associations with lower levels of progesterone, indolelactic acid, hexenoylcarnitine (C6:1), hexadecenoylcarnitine (C16:1) and DHA. Indolelactic acid is a tryptophan catabolite that has an important role in the pathophysiology of obesity $[46,47]$ and is produced entirely by gut microbes [48]. Hexadecenoylcarnitine (C16:1) levels in the blood have been associated with obesity in children [49], while positive effects of DHA on obesity risk and metabolic health have been noted by multiple studies $[50,51]$, with proposed mechanisms including suppression of fatty-acid synthesis, enhancement of fatty-acid $\beta$-oxidation and increase of the serum adiponectin level [52]. The relatively small overlap in cord blood metabolites associated with birthweight and with rapid growth and with obesity, suggests that different mechanisms underlie these outcomes. Furthermore, despite the established association with rapid growth in infancy and later development of overweight, the different directions of effect in birthweight-related metabolites, observed with these two outcomes, suggest different contributory processes. Indeed, lower birthweight was a strong predictor of rapid growth while there was a trend for larger birthweight being associated with overweight in childhood.

Our analysis using a Random Forest classification model revealed that the coupling of the strongly associated molecules and demographic and clinical factors has a high ability to predict overweight/obesity in early childhood. Isganaitis, Rifas-Shiman et al. [18] suggested that cord blood metabolic signatures could be associated with early childhood obesity trajectories demonstrating, in a similar way with our analysis, that prediction models based on prenatal obesity factors (maternal age, pre-pregnancy $\mathrm{BMI}$ and breastfeeding duration) can be improved by adding cord blood associated metabolites. Although models would need to be validated in cohorts that are independent of the selection of metabolites, our results highlight a potential practical application of metabolomics to identify children at risk of obesity and support the potential merit of routine screening of cord blood [53].

A strength of our study includes the use of cord blood from multiple birth cohorts, enabling assessment of the metabolome prior to infant growth, limiting reverse causality. We included a number of prenatal sociodemographic and clinical factors in our analysis. However, we did not have complete data related to maternal nutrition and physical activity that could be linked to both the metabolome and the family environment later in life. Nevertheless, we used paternal socioeconomic factors and maternal clinical factors such as BMI that can reflect general patterns of family nutrition [54] and physical activity [55-57]. Future studies, with high-quality dietary data available, should explore the role of maternal nutrition on the cord blood metabolome.

Although the samples were analysed within a single analytical run in random order, we observed heterogeneity across the cohort metabolomic signatures, mainly explained by the processing of cord blood into plasma or serum. This heterogeneity can influence the observed associations, and for this reason, we added 
in the model a random effect variable for the cohort. Another limitation was that the sample was selected from the general population and we, therefore, had a relatively low number of overweight children. Furthermore, the use of BMI $z$ scores to classify children as overweight is a blunter assessment of adiposity than direct measures such as dual-energy X-ray absorptiometry [58]. We used WHO obesity classification criteria, which have higher sensitivity and lower specificity in identifying obese subjects than the International Obesity Task Force cutoffs. The untargeted approach is both a strength and limitation: while it provides wide metabolome coverage [59], identification of the features can be challenging. Indeed, we were also unable to characterise all the significant features in the MWAS analysis.

\section{CONCLUSION}

We have demonstrated metabolic profiles associated with rapid growth in infancy and overweight/obesity in early childhood, highlighting the role of multiple metabolites in various pathways. We presented evidence that cholestenone and BCAAs are associated with rapid growth in infancy and overweight/obesity in early childhood, respectively, and provide new insights on the potential mechanism underlying overweight risk, particularly early in development. Our findings present a potential route to the identification of at-risk children for the provision of targeted interventions to improve outcomes for children living in obesogenic environments.

\section{REFERENCES}

1. Abarca-Gómez L, Abdeen ZA, Hamid ZA, Abu-Rmeileh NM, Acosta-Cazares B, Acuin $C$, et al. Worldwide trends in body-mass index, underweight, overweight, and obesity from 1975 to 2016: a pooled analysis of 2416 population-based measurement studies in 128.9 million children, adolescents, and adults. Lancet. 2017;390:2627-42.

2. Segal $A B$, Huerta $M C$, Aurino $E$, Sassi F. The impact of childhood obesity on human capital in high-income countries: a systematic review. Obes Rev. 2020;22:e13104.

3. Liu J-H, Jones SJ, Sun H, Probst JC, Merchant AT, Cavicchia P. Diet, physical activity, and sedentary behaviors as risk factors for childhood obesity: an urban and rural comparison. Child Obes. 2012;8:440-8.

4. Patrick K, Norman GJ, Calfas KJ, Sallis JF, Zabinski MF, Rupp J, et al. Diet, physical activity, and sedentary behaviors as risk factors for overweight in adolescence. Arch Pediatr Adoles Med. 2004;158:385-90.

5. Demetriou CA, van Veldhoven K, Relton C, Stringhini S, Kyriacou K, Vineis $P$. Biological embedding of early-life exposures and disease risk in humans: a role for DNA methylation. Eur J Clin Invest. 2015;45:303-32.

6. Barker DJ, Osmond C. Infant mortality, childhood nutrition, and ischaemic heart disease in England and Wales. Lancet. 1986;327:1077-81.

7. Godfrey KM. Maternal regulation of fetal development and health in adult life. Eur J Obstet Gynecol Reprod Biol. 1998;78:141-50.

8. Huang JS, Lee TA, Lu MC. Prenatal programming of childhood overweight and obesity. Matern Child Health J. 2007;11:461-73.

9. Yu Z, Han S, Zhu J, Sun X, Ji C, Guo X. Pre-pregnancy body mass index in relation to infant birth weight and offspring overweight/obesity: a systematic review and meta-analysis. PloS ONE. 2013;8:e61627.

10. Oken E, Levitan E, Gillman M. Maternal smoking during pregnancy and child overweight: systematic review and meta-analysis. Int J Obes. 2008;32:201-10.

11. Chen A, Pennell ML, Klebanoff MA, Rogan WJ, Longnecker MP. Maternal smoking during pregnancy in relation to child overweight: follow-up to age 8 years. Int J Epidemiol. 2006;35:121-30.

12. Vafeiadi M, Roumeliotaki T, Myridakis A, Chalkiadaki G, Fthenou E, Dermitzaki $E_{,}$ et al. Association of early life exposure to bisphenol A with obesity and cardiometabolic traits in childhood. Environ Res. 2016;146:379-87.

13. Mao G, Nachman RM, Sun Q, Zhang X, Koehler K, Chen Z, et al. Individual and joint effects of early-life ambient PM 2.5 exposure and maternal prepregnancy obesity on childhood overweight or obesity. Environ Health Perspect. 2017;125:067005.

14. Kim JT, Lee HK. Metabolic syndrome and the environmental pollutants from mitochondrial perspectives. Rev Endocr Metab Disord. 2014;15:253-62.

15. Leal-Witt M, Ramon-Krauel M, Samino S, Llobet M, Cuadras D, Jimenez-Chillaron J, et al. Untargeted metabolomics identifies a plasma sphingolipid-related signature associated with lifestyle intervention in prepubertal children with obesity. Int J Obes. 2018;42:72-78.
16. Lau C-HE, Siskos AP, Maitre L, Robinson O, Athersuch TJ, Want EJ, et al. Determinants of the urinary and serum metabolome in children from six European populations. BMC Med. 2018;16:202.

17. Handakas $E$, Keski-Rahkonen $P$, Chatzi L, Alfano R, Roumeliotaki T, Plusquiz M et al. Cord blood metabolic signatures predictive of childhood overweight and rapid growth. Obes Rev. Under review.

18. Isganaitis E, Rifas-Shiman SL, Oken E, Dreyfuss J, Gall W, Gillman MW, et al. Associations of cord blood metabolites with early childhood obesity risk. Int J Obes. 2015;39:1041-8.

19. Sorrow P, Maguire R, Murphy SK, Belcher SM, Hoyo C. Elevated metabolites of acetaminophen in cord blood of children with obesity. Pediatr Obes. 2019;14: e12465.

20. Hellmuth C, Uhl O, Standl M, Demmelmair H, Heinrich J, Koletzko B, et al. Cord blood metabolome is highly associated with birth weight, but less predictive for later weight development. Obes Facts. 2017;10:85-100.

21. Janssen BG, Madhloum N, Gyselaers W, Bijnens E, Clemente DB, Cox B, et al. Cohort profile: the ENVIRonmental influence ON early AGEing (ENVIR ON AGE): a birth cohort study. Int J Epidemiol. 2017;46:1386-1387m.

22. Guxens M, Ballester F, Espada M, Fernández MF, Grimalt JO, Ibarluzea J, et al. Cohort profile: the INMA-INfancia y Medio Ambiente-(environment and childhood) project. Int J Epidemiol. 2011;41:930-40.

23. Farchi S, Forastiere F, Vecchi Brumatti L, Alviti S, Arnofi A, Bernardini T, et al. Piccolipiù, a multicenter birth cohort in Italy: protocol of the study. BMC Pediatr. 2014;14:36-36.

24. Chatzi L, Leventakou V, Vafeiadi M, Koutra K, Roumeliotaki T, Chalkiadaki G, et al. Cohort profile: the mother-child cohort in Crete. Greece (Rhea study). 2017;46:1392-1393k.

25. Ong KK, Ahmed ML, Emmett PM, Preece MA, Dunger DBJB. Association between postnatal catch-up growth and obesity in childhood. prospective cohort study. 2000;320:967-71.

26. GROUP WMGRS, de Onis M. WHO Child Growth Standards based on length/ height, weight and age. Acta Paediatr. 2006;95:76-85.

27. Royston $P$, Wright EM. A method for estimating age-specific reference intervals ('normal ranges') based on fractional polynomials and exponential transformation. J R Statist Soc. 1998;161:79-101.

28. World Health Organization. WHO child growth standards: training course on child growth assessment. 2008.

29. De Onis M. WHO child growth standards. Geneva: WHO 2006: 1 Á336.

30. De Onis $M$, Lobstein T. Defining obesity risk status in the general childhood population: which cut-offs should we use? In: Taylor \& Francis, 2010.

31. Bates D, Maechler M, Bolker B, Walker S. Ime4: Linear mixed-effects models using Eigen and S4. R package version 2014;1:1-23.

32. Benjamini Y, YJJotRsssB Hochberg. Controlling the false discovery rate: a practical and powerful approach to multiple testing. J R Statist Soc. 1995;57:289-300.

33. Li S, Park Y, Duraisingham S, Strobel FH, Khan N, Soltow QA, et al. Predicting network activity from high throughput metabolomics. PLoS Comput Biol. 2013;9: e1003123.

34. Robinson O, Keski-Rahkonen $P$, Chatzi L, Kogevinas M, Nawrot T, Pizzi C, et al. Cord blood metabolic signatures of birth weight: a population-based study. J Proteome Res. 2018;17:1235-47.

35. Alfano R, Chadeau-Hyam M, Ghantous A, Keski-Rahkonen P, Chatzi L, Perez AE, et al. A multi-omic analysis of birthweight in newborn cord blood reveals new underlying mechanisms related to cholesterol metabolism. Metabolism. 2020;110:154292.

36. Breiman L. Random forests. Mach Learn. 2001;45:5-32.

37. Suzuki K, Shimizu T, Nakata T. The cholesterol metabolite cholest-4-en-3-one and its 3-oxo derivatives suppress body weight gain, body fat accumulation and serum lipid concentration in mice. Bioorg Med Chem Lett. 1998;8:2133-8.

38. Degenhart $H$, Alsema $G$, Visser $H$. Effects of cholestenone feeding in rats. Pediatr Res. 1981;15:78-78.

39. Kenny DJ, Plichta DR, Shungin D, Koppel N, Hall AB, Fu B, et al. Cholesterol metabolism by uncultured human gut bacteria influences host cholesterol level. Cell Host Microbe. 2020;28:245-257.e6.

40. Moore RE, Townsend SD. Temporal development of the infant gut microbiome. Open Biol. 2019;9:190128.

41. Cong X, Xu W, Janton S, Henderson WA, Matson A, McGrath JM, et al. Gut microbiome developmental patterns in early life of preterm infants: impacts of feeding and gender. PloS ONE. 2016;11:e0152751.

42. Groer MW, Luciano AA, Dishaw LJ, Ashmeade TL, Miller E, Gilbert JA. Development of the preterm infant gut microbiome: a research priority. Microbiome. 2014;2:38.

43. Kalliomäki $M$, Collado $M C$, Salminen S, Isolauri E. Early differences in fecal microbiota composition in children may predict overweight. Am J Clin Nutr. 2008;87:534-8.

44. Lynch CJ, Adams SH. Branched-chain amino acids in metabolic signalling and insulin resistance. Nat Rev Endocrinol. 2014;10:723-36. 
45. Clandinin MT, Van Aerde JE, Merkel KL, Harris CL, Springer MA, Hansen JW, et al. Growth and development of preterm infants fed infant formulas containing docosahexaenoic acid and arachidonic acid. J Pediatr. 2005;146:461-8.

46. Sun L, Ma L, Ma Y, Zhang F, Zhao C, Nie Y. Insights into the role of gut microbiota in obesity: pathogenesis, mechanisms, and therapeutic perspectives. Protein Cell. 2018;9:397-403.

47. Ni Y, Ni L, Zhuge F, Fu Z. The gut microbiota and its metabolites, novel targets for treating and preventing non-alcoholic fatty liver disease. Mol Nutr Food Res. 2020;64:2000375.

48. Agus A, Planchais J, Sokol H. Gut microbiota regulation of tryptophan metabolism in health and disease. Cell Host Microbe. 2018;23:716-24.

49. Wahl S, Yu Z, Kleber M, Singmann P, Holzapfel C, He Y, et al. Childhood obesity is associated with changes in the serum metabolite profile. Obes Facts. 2012;5:660-70.

50. Simopoulos AP. An increase in the omega-6/omega-3 fatty acid ratio increases the risk for obesity. Nutrients. 2016;8:128.

51. Kunešová $M$, Braunerova R, Hlavatý $P$, Tvrzicka E, Staňková B, Škrha J et al. The influence of $n-3$ polyunsaturated fatty acids and very low calorie diet during a short-term weight reducing regimen on weight loss and serum fatty acid composition in severely obese women. Physiol Res. 2006;55:63-72.

52. Shirouchi B, Nagao K, Inoue N, Ohkubo T, Hibino H, Yanagita T. Effect of dietary omega 3 phosphatidylcholine on obesity-related disorders in obese Otsuka LongEvans Tokushima fatty rats. J Agric Food Chem. 2007;55:7170-6.

53. Kelly N, Makarem DC, Wasserstein MP. Screening of newborns for disorders with high benefit-risk ratios should be mandatory. J Law Med Ethics. 2016;44:231-40.

54. Williams JE, Helsel B, Griffin SF, Liang J. Associations between parental BMI and the family nutrition and physical activity environment in a community sample. J Commun Health. 2017;42:1233-9.

55. McMurray RG, Harrell JS, Deng S, Bradley CB, Cox LM, Bangdiwala SI. The influence of physical activity, socioeconomic status, and ethnicity on the weight status of adolescents. Obes Res. 2000;8:130-9.

56. Yang XL, Telama R, Laakso L. Parents' physical activity, socioeconomic status and education as predictors of physical activity and sport among children and youths-A 12-year follow-up study. Int Rev Soc Sport. 1996;31:273-91.

57. Lampinen E-K, Eloranta A-M, Haapala EA, Lindi V, Väistö J, Lintu N, et al. Physical activity, sedentary behaviour, and socioeconomic status among Finnish girls and boys aged 6-8 years. Eur J Sport Sci. 2017;17:462-72.

58. Freedman DS, Sherry B. The validity of BMI as an indicator of body fatness and risk among children. Pediatrics. 2009;124:S23-S34.

59. Sun YV, Hu Y-J. Integrative analysis of multi-omics data for discovery and functional studies of complex human diseases. Adv Genet. 2016;93:147-90.

\section{ACKNOWLEDGEMENTS}

This research was supported by European Commission Horizon 2020 Grant to the 'STOP Project' (Grant ref. 774548). This study was funded by the European Union Social Fund and the Hellenic Ministry of Health ("Programme of prevention and early diagnosis of obesity and neurodevelopment disorders in preschool-age children in the prefecture of Heraklion, Crete, Greece' MIS number 349580, NSRF 2007-2013). Additional funding from the National Institute of Environmental Health Sciences (NIEHS) supported Dr. Chatzi (R01ES030691, R01ES029944, R01ES030364, R21ES029681, R21ES028903 and P30ES007048). The ENVIRONAGE birth-cohort is supported by the EU Program 'Ideas' (ERC-2012-StG-310898) and the FWO $(\mathrm{G} 082317 \mathrm{~N})$, a PhD fellowship of the Bijzonder Onderzoeksfonds (BOF) at Hasselt University supported MD Alfano. We acknowledge support from the Spanish Ministry of Science, Innovation and Universities, 'Centro de Excelencia Severo Ochoa 2013-
2017", SEV-2012-0208 and 'Secretaria d'Universitats i Recerca del Departament d'Economia i Coneixement de la Generalitat de Catalunya' (2017SGR595). O.R. was supported by a UKRI Future Leaders Fellowship (MR/S03532X/1).

\section{AUTHOR CONTRIBUTIONS}

O.R., L.C. and P.V. conceived the study. E.H. performed most statistical analyses. E.H. and O.R. draughted the manuscript. P.K.R., N.R. and A.S. acquired the MSy data and conducted structural annotation. T.R. contributed additional analyses for age prediction models. M.P. and T.N. coordinated data/sample collection in ENVIRONAGE, S.B. and L.R. coordinated data/sample collection in Piccolipiu, L.M. and M.V. coordinated data/sample collection in INMA, T.R. and L.C. coordinated data/sample collection in Rhea, R.A., E.H. and O.R. prepared and supervised data collection and curation. O.R. coordinated and supervised. All authors critically reviewed the manuscript.

\section{COMPETING INTERESTS}

The authors declare no competing interests. Where authors are identified as personnel of the International Agency for Research on Cancer/World Health Organisation, the authors alone are responsible for the views expressed in this article and they do not necessarily represent the decisions, policy or views of the International Agency for Research on Cancer/World Health Organisation.

\section{ADDITIONAL INFORMATION}

Supplementary information The online version contains supplementary material available at https://doi.org/10.1038/s41366-021-00888-1.

Correspondence and requests for materials should be addressed to O.R.

Reprints and permission information is available at http://www.nature.com/ reprints

Publisher's note Springer Nature remains neutral with regard to jurisdictional claims in published maps and institutional affiliations.

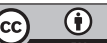

Open Access This article is licensed under a Creative Commons Attribution 4.0 International License, which permits use, sharing, adaptation, distribution and reproduction in any medium or format, as long as you give appropriate credit to the original author(s) and the source, provide a link to the Creative Commons license, and indicate if changes were made. The images or other third party material in this article are included in the article's Creative Commons license, unless indicated otherwise in a credit line to the material. If material is not included in the article's Creative Commons license and your intended use is not permitted by statutory regulation or exceeds the permitted use, you will need to obtain permission directly from the copyright holder. To view a copy of this license, visit http://creativecommons. org/licenses/by/4.0/.

(c) The Author(s) 2021 